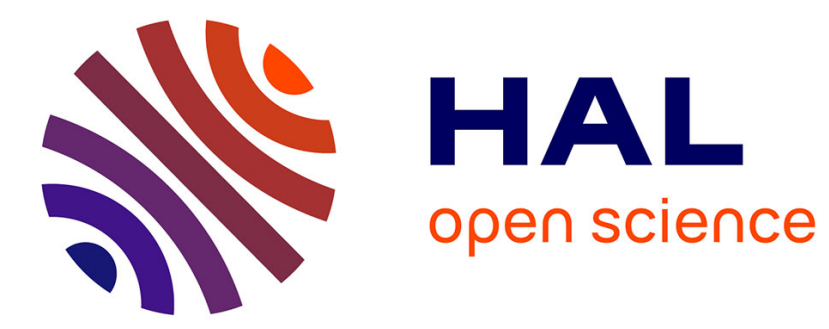

\title{
Fast Minor Testing in Planar Graphs
}

Isolde Adler, Frederic Dorn, Fedor V. Fomin, Ignasi Sau, Dimitrios M. Thilikos

\section{To cite this version:}

Isolde Adler, Frederic Dorn, Fedor V. Fomin, Ignasi Sau, Dimitrios M. Thilikos. Fast Minor Testing in Planar Graphs. ESA 2010 - 18th Annual European Symposium on Algorithms, Sep 2010, Liverpool, United Kingdom. pp.97-109, 10.1007/978-3-642-15775-2_9 . lirmm-00736769

\section{HAL Id: lirmm-00736769 https://hal-lirmm.ccsd.cnrs.fr/lirmm-00736769}

Submitted on 16 Sep 2019

HAL is a multi-disciplinary open access archive for the deposit and dissemination of scientific research documents, whether they are published or not. The documents may come from teaching and research institutions in France or abroad, or from public or private research centers.
L'archive ouverte pluridisciplinaire HAL, est destinée au dépôt et à la diffusion de documents scientifiques de niveau recherche, publiés ou non, émanant des établissements d'enseignement et de recherche français ou étrangers, des laboratoires publics ou privés. 


\title{
Fast Minor Testing in Planar Graphs
}

\author{
Isolde Adler ${ }^{1}$, Frederic Dorn ${ }^{2}$, Fedor V. Fomin ${ }^{2}$, \\ Ignasi $\mathrm{Sau}^{3}$, and Dimitrios M. Thilikos ${ }^{4, \star}$ \\ 1 Institut für Informatik, Goethe-Universität, Frankfurt, Germany \\ iadler@informatik. uni-frankfurt.de \\ 2 Department of Informatics, University of Bergen, Norway \\ \{frederic.dorn,fedor.fomin\}@ii.uib.no \\ 3 Department of Computer Science, Technion, Haifa, Israel \\ ignasi@cs.technion.ac.il \\ 4 Department of Mathematics, National and Kapodistrian \\ University of Athens, Greece \\ sedthilk@math.uoa.gr
}

\begin{abstract}
Minor containment is a fundamental problem in Algorithmic Graph Theory, as numerous graph algorithms use it as a subroutine. A model of a graph $H$ in a graph $G$ is a set of disjoint connected subgraphs of $G$ indexed by the vertices of $H$, such that if $\{u, v\}$ is an edge of $H$, then there is an edge of $G$ between components $C_{u}$ and $C_{v}$. Graph $H$ is a minor of $G$ if $G$ contains a model of $H$ as a subgraph. We give an algorithm that, given a planar $n$-vertex graph $G$ and an $h$-vertex graph $H$, either finds in time $2^{\mathcal{O}(h)} \cdot n+\mathcal{O}\left(n^{2} \cdot \log n\right)$ a model of $H$ in $G$, or correctly concludes that $G$ does not contain $H$ as a minor. Our algorithm is the first singleexponential algorithm for this problem and improves all previous minor testing algorithms in planar graphs. Our technique is based on a novel approach called partially embedded dynamic programming.
\end{abstract}

Keywords: graph minors, planar graphs, branchwidth, parameterized complexity, dynamic programming.

\section{Introduction}

For two input graphs $G$ and $H$, the Minor Containment problem is to decide whether $H$ is a minor of $G$. This is a classical NP-complete problem [16, and remains NP-complete even when both graphs $G$ and $H$ are planar, as it is a generalization of the HAMILTONIAN CYClE problem. When $H$ is fixed, by the celebrated result of Robertson and Seymour [26, there is an algorithm to decide if $H$ is a minor of an input graph $G$ that runs in time $f(h) \cdot n^{3}$, where $n$ is the number of vertices of $G, h$ is the number of vertices in $H$, and $f$ is some recursive function. One of the significant algorithmic implications of this result is that, combined with the Graph Minor Theorem of Robertson and Seymour 28, it shows the polynomial-time solvability of many graph problems, some of which

\footnotetext{
* Supported by the project "Kapodistrias" (A $\Pi$ 02839/ 28.07.2008) of the National and Kapodistrian University of Athens (project code: 70/ 4/8757.)
} 
were previously not even known to be decidable [15. However, these algorithmic results are highly non-practical. This triggered an ongoing quest in the Theory of Algorithms since then for making Graph Minors constructive and for making its algorithmic proofs practical for a wide range of applications (e.g., 8, 20]).

Unfortunately, in the minor testing algorithm of Robertson and Seymour [26], the function $f(h)$ has an immense exponential growth, which makes the algorithm absolutely impractical even for very simple patterns (see 21 for recent theoretical improvements of this function). There were several attempts to improve the running time of the algorithm of Robertson and Seymour. One direction of such improvements is decreasing the degree of the polynomial in $n$. For example, Reed and Li gave a linear time algorithm solving $K_{5}$-minor containment 25]. The second direction of improvements is towards reducing the exponential dependency in the function $f(h)$, which is a natural direction of study for Parameterized Complexity 14. A significant step in this direction was done by Hicks [19], who provided in graphs of branchwidth $k$ and $m$ edges an $\mathcal{O}\left(3^{k^{2}} \cdot(h+k-1) ! \cdot m\right)$ time algorithm, following the algorithm sketched by Robertson and Seymour [26]. Recently, this was improved to $\mathcal{O}\left(2^{(2 k+1) \log k} \cdot h^{2 k} \cdot 2^{2 h^{2}} \cdot m\right)$ on general graphs, and in planar, and more generally, in graphs of bounded genus, to $2^{\mathcal{O}(k)} \cdot h^{2 k} \cdot 2^{\mathcal{O}(h)} \cdot n[1]$.

In this paper we focus on the case where the input graph $G$ is planar.

Planar $H$-Minor Containment

Input: A planar graph $G$.

Objective: Either find a model $M$ of $H$ in $G$, or conclude that $G$ does not contain such a model.

By arguments inspired by Bidimensionality Theory [5], it can be shown that the $2^{\mathcal{O}(k)} \cdot h^{2 k} \cdot 2^{\mathcal{O}(h)} \cdot n$ time algorithm from [1], combined with the grid minor Theorem of Robertson, Thomas, and Seymour [27, can be used to solve PLANAR $H$-Minor Containment in time $2^{\mathcal{O}(h \log h)} \cdot n+\mathcal{O}\left(n^{2} \cdot \log n\right)$. This directly sets up the challenge of designing a single-exponential (on the size $h$ of the pattern $H$ ) algorithm for this problem. Over the last four decades, many different algorithmic techniques in planar graphs were developed for different type of problems and algorithms, including approximation [4,7], exact [12, 22, and parameterized algorithms $3,6,13$. However, it seems that none of these approaches can be used to speed up the algorithm for Planar $H$-Minor Containment.

Our results and key ideas. Our main result is the following theorem.

Theorem 1. Given a planar graph $G$ on $n$ vertices and a graph $H$ on $h$ vertices, we can solve Planar $H$-Minor Containment in time $2^{\mathcal{O}(h)} \cdot n+\mathcal{O}\left(n^{2} \cdot \log n\right)$.

That is, we prove that when $G$ is planar the behavior of the function $f(h)$ can be made single-exponential, improving over all previous results for this problem [1, 26, 19. In addition, we can enumerate and count the number of models within the same time bounds. Let us remark that by our theorem, PLANAR $H$-MinOR 
Containment is solvable in polynomial time when the size of the pattern $H$ is $\mathcal{O}(\log n)$, substantially improving the existing algorithms for small patterns [10].

In order to prove Theorem 11. we introduce a novel approach of dynamic programming in planar graphs of bounded branchwidth, namely partially embedded dynamic programming. This approach is extremely helpful in computing graph minors but we believe that this technique can be used in many related problems including Planar Disjoint Paths. Our technique is inspired by the technique of embedded dynamic programming introduced in [11] for solving PLANAR SuBGRAPH ISOMORPHISM for a pattern of size $h$ and an input graph of size $n$ in time $2^{\mathcal{O}(h)} \cdot n$. There, one controls the partial solutions by the ways the separators of $G$ can be routed through the pattern. The difference (and difficulty) concerning Planar $H$-Minor Containment is that we look for a model $M$ of size $\mathcal{O}(n)$ out of $2^{\mathcal{O}(n)}$ possible non-isomorphic models of $H$ in $G$. In partially embedded dynamic programming, we look for potential models of $H$ in $G$ with a magnifying glass only at a given separator $S$ of $G$. That is, we consider a collection $\mathcal{A}$ of graphs $A$ arising from 'inflating' a part of $H$, namely the part interacting with $S$. Thus, each $A$ behaves like a subgraph of $G$ inside the intersection with $S$, and outside that intersection $A$ behaves like a minor of $G$; this is why we call our dynamic programming technique 'partially embedded'.

After giving some preliminaries in Section 2, we first show in Section 3 how Planar $H$-Minor Containment can be solved in polynomial time for input graphs of large branchwidth (in comparison to the pattern size). If the branchwidth is small, we compute the collection $\mathcal{A}$ in Section 3.1 and give the partially embedded dynamic programming approach in Section 3.2 .

\section{Preliminaries}

Graphs and graph minors. We use standard graph terminology, see for instance [9]. All graphs considered in this article are simple and undirected. Given a graph $G$, we denote by $V(G)$ and $E(G)$ the vertex set and the edge set of $G$, respectively. A graph $H$ is a subgraph of a graph $G, H \subseteq G$, if $V(H) \subseteq V(G)$ and $E(H) \subseteq E(G)$. We define graph operation contracting edge $e=\{x, y\} \in G$ by removing $e$, including $x$ and $y$, and making a new vertex $v_{e}$ adjacent to the former neighbors of $x$ and $y$ (excluding $x$ and $y$ ).

Graph $H$ is a minor of graph $G$ (denoted by $H \preceq G$ ), if $H$ can be obtained from a subgraph of $G$ by a (possibly empty) sequence of edge contractions. In this case we also say that $G$ is a major of $H$. Graph $H$ is a contraction minor of graph $G$ (denoted by $H \preceq_{c} G$ ), if $H$ can be obtained from $G$ by a (possibly empty) sequence of edge contractions.

A model of $H$ in $G$ [26] is a mapping $\phi$, that assigns to every edge $e \in E(H)$ an edge $\phi(e) \in E(G)$, and to every vertex $v \in V(H)$ a non-empty connected subgraph $\phi(v) \subseteq G$, such that

(i) the graphs $\{\phi(v) \mid v \in V(H)\}$ are mutually vertex-disjoint and the edges $\{\phi(e) \mid e \in E(H)\}$ are pairwise distinct; and

(ii) for $e=\{u, v\} \in E(H), \phi(e)$ connects $\phi(u)$ with $\phi(v)$. 
Thus, $H$ is isomorphic to a minor of $G$ if and only if there exists a model of $H$ in $G$.

With slight abuse of notation, the subgraph $M \subseteq G$ defined by the union of $\{\phi(v) \mid v \in V(H)\}$ and $\{\phi(e) \mid e \in E(H)\}$ is also called a model of $H$ in $G$. The edge set of a model $M$ is partitioned into c-edges (contraction edges) and m-edges (minor edges), which are the edges of $\{\phi(v) \mid v \in V(H)\}$ and $\{\phi(e) \mid e \in E(H)\}$, respectively.

Branchwidth. A branch decomposition $(T, \mu)$ of a graph $G$ consists of an unrooted ternary tree $T$ (i.e., all internal vertices are of degree three) and a bijection $\mu: L \rightarrow E(G)$ from the set $L$ of leaves of $T$ to the edge set of $G$. We define for every edge $e$ of $T$ the middle set $\operatorname{mid}(e) \subseteq V(G)$ as follows: Let $T_{1}$ and $T_{2}$ be the two connected components of $T \backslash\{e\}$. Then let $G_{i}$ be the graph induced by the edge set $\left\{\mu(f): f \in L \cap V\left(T_{i}\right)\right\}$ for $i \in\{1,2\}$. The middle set is the intersection of the vertex sets of $G_{1}$ and $G_{2}$, i.e., $\operatorname{mid}(e)=V\left(G_{1}\right) \cap V\left(G_{2}\right)$. The width of $(T, \mu)$ is the maximum order of the middle sets over all edges of $T$, i.e., width $(T, \mu):=\max \{|\operatorname{mid}(e)|: e \in E(T)\}$. The branchwidth of $G$ is defined as $\operatorname{bw}(G):=\min \{\operatorname{width}(T, \mu) \mid(T, \mu)$ branch decomposition of $G\}$. Note that for each $e \in E(T), \operatorname{mid}(e)$ is a separator of $G$, unless $\operatorname{mid}(e)=\emptyset$.

Remark 1. For every two edges $e, f \in E(T)$ with $e \cap f \neq \emptyset$, we have $\mid \operatorname{mid}(e) \cup$ $\operatorname{mid}(f) \mid \leq 1.5 \cdot \operatorname{width}(T, \mu)$.

Intuitively, a graph $G$ has small branchwidth if $G$ is close to being a tree. The fundamental grid minor Theorem says that, roughly, a graph has either small branchwidth, or it contains a large grid as a minor. We use the variant for planar graphs.

Proposition $1([\mathbf{2 7}, \mathbf{1 8}])$. Given a planar graph $G$ on $n$ vertices with $\mathbf{b w}(G) \geq$ $k$, a model of the $(\lfloor k / 3\rfloor \times\lfloor k / 3\rfloor)$-grid in $G$ can be found in time $\mathcal{O}\left(n^{2} \cdot \log n\right)$.

On the other hand, every planar graph is minor of a large enough grid.

Proposition 2 ([27]). If $H$ is a planar graph with $|V(H)|+2|E(H)| \leq \ell$, then $H$ is isomorphic to a minor of the $(2 \ell \times 2 \ell)$-grid.

Planar graphs and equivalent drawings. Let $\Sigma$ be the unit sphere. A planar drawing, or simply drawing, of a graph $G$ with vertex set $V(G)$ and edge set $E(G)$ maps vertices to points in the sphere, and edges to simple curves between their end-vertices, such that edges do not cross, except in common end-vertices. A plane graph is a graph $\mathrm{G}$ together with a planar drawing. A planar graph is a graph that admits a planar drawing. The set of faces $F(G)$ of a plane graph $G$ is defined as the union of the connected regions of $\Sigma \backslash G$. A subgraph of a plane graph $G$, induced by the vertices and edges incident to a face $f \in F(G)$, is called a bound of $f$ (for further reading, see e.g. [9]). Consider any two drawings $G_{1}$ and $G_{2}$ of a planar graph $G$. A homeomorphism of $G_{1}$ onto $G_{2}$ is a homeomorphism of $\Sigma$ onto itself which maps vertices, edges, and faces of $G_{1}$ onto vertices, edges, and faces of $G_{2}$, respectively. We call two planar drawings equivalent, if there is a homeomorphism from one onto the other. 
Proposition 3 (e.g. [24]). The number of non-equivalent drawings of a planar $n$-vertex graph is $2^{\mathcal{O}(n)}$. A set of all such drawings can be computed in time $2^{\mathcal{O}(n)}$.

Proposition 4 ([31]). The number of non-isomorphic edge-maximal planar $n$ vertex graphs is $2^{\mathcal{O}(n)}$.

Nooses and combinatorial nooses. A noose of a $\Sigma$-plane graph $G$ is a simple closed curve in $\Sigma$ that meets $G$ only in vertices. From the Jordan Curve Theorem (e.g. 23]), it follows that nooses separate $\Sigma$ into two regions.

Let $V(N)=N \cap V(G)$ be the vertices and $F(N)$ be the faces intersected by a noose $N$. The length of $N$ is $|V(N)|$, the number of vertices in $V(N)$. The clockwise order in which $N$ meets the vertices of $V(N)$ is a cyclic permutation $\pi$ on the set $V(N)$.

A combinatorial noose $N_{C}=\left[v_{0}, f_{0}, v_{1}, f_{1}, \ldots, f_{\ell-1}, v_{\ell}\right]$ in a plane graph $G$ is an alternating sequence of vertices and faces of $G$, such that

- $f_{i}$ is a face incident to both $v_{i}, v_{i+1}$ for all $i<\ell$;

- $v_{0}=v_{\ell}$ and the vertices $v_{1}, \ldots, v_{\ell}$ are mutually distinct; and

- if $f_{i}=f_{j}$ for any $i \neq j$ and $i, j=0, \ldots, \ell-1$, then the vertices $v_{i}, v_{i+1}, v_{j}$, and $v_{j+1}$ do not appear in the order $\left(v_{i}, v_{j}, v_{i+1}, v_{j+1}\right)$ on the bound of face $f_{i}=f_{j}$.

The length of a combinatorial noose $\left[v_{0}, f_{0}, v_{1}, f_{1}, \ldots, f_{\ell-1}, v_{\ell}\right]$ is $\ell$.

Remark 2. The order in which a noose $N$ intersects the faces $F(N)$ and the vertices $V(N)$ of a plane graph $G$ gives a unique alternating face-vertex sequence of $F(N) \cup V(N)$ which is a combinatorial noose $N_{C}$. Conversely, for every combinatorial noose $N_{C}$ there exists a noose $N$ with face-vertex sequence $N_{C}$.

We will refer to combinatorial nooses simply as nooses if it is clear from the context.

Proposition 5 ([1] $)$. Every plane graph on $n$ vertices has $2^{\mathcal{O}(n)}$ combinatorial nooses.

Sphere cut decompositions. For a plane graph $G$, we define a sphere cut decomposition (or sc-decomposition) $\langle T, \mu, \pi\rangle$ as a branch decomposition, which for every edge $e$ of $T$ has a noose $N_{e}$ that divides $\Sigma$ into two regions $\Delta_{1}$ and $\Delta_{2}$ such that $G_{i} \subseteq \Delta_{i} \cup N_{e}$, where $G_{i}$ is the graph induced by the edge set $\{\mu(f): f \in$ $\left.L \cap V\left(T_{i}\right)\right\}$ for $i \in\{1,2\}$ and $T_{1} \dot{\cup} T_{2}=T \backslash\{e\}$. Thus $N_{e}$ meets $G$ only in $V\left(N_{e}\right)=$ $\operatorname{mid}(e)$ and its length is $|\operatorname{mid}(e)|$. The vertices of $\operatorname{mid}(e)=V\left(G_{1}\right) \cap V\left(G_{2}\right)$ are ordered according to a cyclic permutation $\pi=\left(\pi_{e}\right)_{e \in E(T)}$ on $\operatorname{mid}(e)$.

Theorem 2 ( [13, 30]). Let $G$ be a planar graph of branchwidth at most $k$ without vertices of degree one embedded on a sphere. Then there exists a scdecomposition of $G$ of width at most $k$. 


\section{Minor Testing in Planar Graphs}

For solving Planar $H$-Minor Containment in single-exponential time $2^{\mathcal{O}(h)}$. $n+\mathcal{O}\left(n^{2} \cdot \log n\right)$, we introduce in this section the method of partially embedded dynamic programming. We present Algorithm 1 as a roadmap on how we proceed in proving our main Theorem 1 .

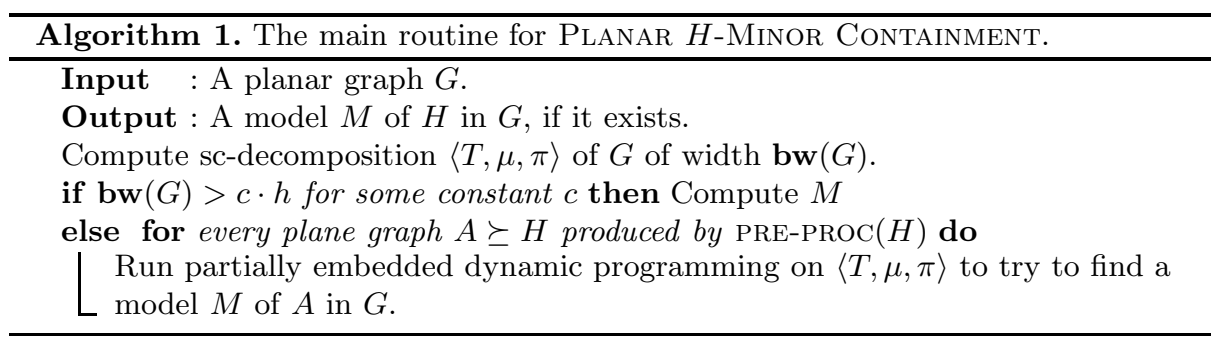

We divide Algorithm 1 into three parts, presented in the following sections.

From the next proposition we can find a model of $H$ in $G$ in the case of $G$ having large branchwidth.

Proposition 6. $[\star]$ Let $G$ and $H$ be planar graphs with $|V(G)|=n,|V(H)|=$ $h$. There exists a small constant $c$ such that if $\mathbf{b w}(G)>c \cdot h$, then $G$ contains a model of $H$, which can be found in time $\mathcal{O}\left(n^{2} \cdot \log n+h^{4}\right)$.

Otherwise, let us assume that $\mathbf{b w}(G) \leq c \cdot h$. In this case, $\operatorname{PrE-PROC}(H)$ (basically) computes a list of all plane majors $A$ of $H$ up to a fixed size linear in $h$. This 'preprocessing step' is presented in Section 3.1. In the sequel, we will only be interested in a graph $A$ of our list, if $A$ is a minor of $G$ obtained from $H$ by 'uncontracting' some part, such that on a given subset $S \subseteq V(G)$, our graph $A$ looks like a subgraph of $G$. Finally, in Section 3.2 , we proceed by partially embedded dynamic programming bottom-up along a sphere cut decomposition of $G$. Here we make use of the fact that every middle set $S$ yields a separating noose in an embedding of $G$. If $H$ has a model $M \subseteq G$ that intersects $S$, then the noose comes from a noose in $M$, which in turn is present in some major $A$ of $H$ of our list. We use this fact to restrict the number of candidates we need to consider.

\subsection{Preprocessing}

If the branchwidth of $G$ is at most $c \cdot h$, then we compute a sphere cut decomposition of width $\mathcal{O}(h)$ in time $\mathcal{O}\left(n^{2}\right)$ by using the algorithm of [17], and we continue with dynamic programming.

In the first step we do preprocessing. Namely, we compute for $H$ a list of auxiliary graphs $A$ with $H \preceq A \preceq M$ and $|V(A)|=\mathcal{O}(h)$, such that $A$ is a

\footnotetext{
${ }^{1}$ The parts marked with $[\star]$ can be found in a longer version of our paper [2].
} 
candidate for a model $M$ in $G$. To be precise, we compute a collection $\mathcal{A}$ of 2-edge-colored plane graphs, each consisting of

- a planar graph $A$ with $|V(A)| \leq h+1.5 \cdot \mathbf{b w}(G)$, such that $H$ is a contraction minor of $A$;

- a bipartition of the edge set $E(A)$ into m-edges and c-edges such that contracting the c-edges of $A_{m, c}$ creates a graph isomorphic to $H$; and

- a drawing $\Phi$ of $A_{m, c}$.

The simple routine PRE-PROC $[\star]$ takes as input $H$ and outputs the collection $\mathcal{A}$ of 2-edge-colored plane graphs $\left\langle A_{m, c}, \Phi\right\rangle$.

When doing dynamic programming in Section 3.2, instead of finding a model $M$ of $H$ in $G$, we restrict ourselves to finding such a collection $\mathcal{A}$ consisting of minors of $M$ which represent both $H$ and $M$ in each dynamic programming step.

Lemma 1. $[\star]$ For every planar graph $H$ on $h$ vertices and every constant $d$, the cardinality of the collection $\mathcal{A}$ of non-isomorphic 2-edge-colored plane graphs on $d \cdot h$ vertices containing a minor isomorphic to $H$ is $2^{\mathcal{O}(h)}$. Furthermore, we can compute $\mathcal{A}$ in time $2^{\mathcal{O}(h)}$.

Using Lemma 1, we get the following corollary.

Corollary 1. Algorithm PRE-PROC $[\star]$ is correct and runs in time $2^{\mathcal{O}(\mathbf{b w}(G)+h)}$.

\subsection{Partially Embedded Dynamic Programming}

From now on, we will refer to a 2-edge-colored plane graph $\left\langle A_{m, c}, \Phi\right\rangle \in \mathcal{A}$ simply as $A$. In this section we present our technique of partially embedded dynamic programming, using it to solve Planar $H$-Minor Containment in the case of the input graph $G$ having bounded branchwidth. Before proceeding to a formal description of the dynamic programming, we provide the basic intuition behind our algorithm. Towards this, let us consider graphs $A \in \mathcal{A}$ satisfying $H \preceq_{c} A$ and $A \preceq G$.

We define subgraphs PASt,PRESEnt, and FUtURE of $A$ with $V(A)=V($ PAST $) \cup$ $V(\mathrm{PRESENT}) \cup V(\mathrm{FutuRE})$ and $E(A)=E(\mathrm{PASt}) \dot{U} E(\mathrm{PRESENT}) \dot{\cup} E(\mathrm{FUtURE})$, such that

- PRESEnt $\subseteq G$, (i.e., we can obtain $A$ as a minor of $G$ with PRESENT being subgraph of $G$ ); and

- $E($ PAST $) \subseteq E(H)$, (i.e., we can obtain $H$ as a contraction minor of $A$ without contracting edges in PAST) 2

Intuitively speaking, in partially embedded dynamic programming, we look for potential models $M$ of $H$ with a magnifying glass only in the separators of the sc-decomposition of $G$. By decontracting $H$ at the separators, we obtain the

${ }^{2}$ Here, we slightly abuse notation by assuming that edge sets in different graphs are actually the same, instead of introducing bijective mappings. Note that we make no assumption about the edges in FUTURE. 
part PRESENT, which yields a subgraph of $G$ for which we are enabled to apply embedded dynamic programming. For memorizing the rest of the potential model $M$, we contract all necessary edges to PAST in the processed graph and (almost) all edges to FUTURE in the graph remainder. The picture will be made clearer in the sequel.

Given a sc-decomposition of $G$, we proceed with dynamic programming: Every edge $e$ of the sc-decomposition defines a separator $\operatorname{mid}(e) \subseteq V(G)$ and an associated noose $N_{e}$, which separates the graph $G_{\text {sub }} \subseteq G$ processed so far from $G \backslash G_{\text {sub }}$. At every edge $e$ of the sc-decomposition, we check for every graph $A$ of $\mathcal{A}$ all the ways in which the graph $G_{\text {sub }}$ can be obtained as a major of $A_{\text {sub }} \subseteq A$ with $A_{\text {sub }}=(V($ PAST $) \cup V($ PRESENT $), E($ PAST $) \cup E($ PRESENT $))$, where $\operatorname{mid}(e)$ determines $V$ (PRESENT). The noose $N_{e}$ comes from a noose in $A$, and this is controlled by the ways in which $N_{e}$ can be routed through the vertices of $A$. The number of solutions we get - the valid partial solutions - is bounded by the number of combinatorial nooses in $A$ onto which we can map $N_{e}$. When updating the valid partial solutions at two incident edges of the sc-decomposition, we unite PRESENT and PAST of two solutions and set the graph remainder to FUTURE. In a post-processing step, we contract part of PRESENT, namely those edges with at most one endpoint in the newly obtained separator of the sc-decomposition; this part becomes PAST. We then decontract some edges of FUTURE for the next updating step. This concludes the informal description of the algorithm.

In the remaining part of this section, we will precisely describe and analyze the dynamic programming routine with which we achieve the following result:

Lemma 2. For a plane graph $G$ with a given sc-decomposition $\langle T, \mu, \pi\rangle$ of $G$ of width $\mathrm{bw}(G)$ and a planar graph $H$ on $h$ vertices, we can decide in time

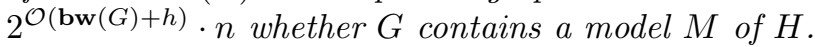

Dynamic programming. We root the sc-decomposition $\langle T, \mu, \pi\rangle$ at some node $r \in V(T)$. For each edge $e \in T$, let $L_{e}$ be the set of leaves of the subtree rooted at $e$. The subgraph $G_{e}$ of $G$ is induced by the edge set $\left\{\mu(v) \mid v \in L_{e}\right\}$. The vertices of $\operatorname{mid}(e)$ form a combinatorial noose $N$ that separates $G_{e}$ from the residual graph.

Let $A$ be a given plane graph in $\mathcal{A}$. If $A$ is a minor of $G$, then there exists a plane model $M$ of $A$ in $G$. Furthermore, for above noose $N$ the intersection $M \cap N$ forms a noose in both model $M$ and $A$. One basic point of partially embedded dynamic programming is to check how the vertices of the combinatorial noose $N$ are mapped to faces and vertices of $A$. For a combinatorial noose $N_{A}$ in $A$, we can map $N$ to $N_{A}$ bounding (clockwise) a unique subgraph $A_{\text {sub }}$ of $A$.

In each step of the algorithm, we compute the solutions for a sub-problem in $G_{e}$, where each solution consists of three parts, namely

- a plane 2-edge-colored graph $A \in \mathcal{A}$;

- a combinatorial noose $N_{A}$ in $A$; and

- a mapping $\gamma$ from combinatorial noose $N$ to $N_{A}$ (defined below).

$N_{A}$ has the properties that $a$ ) it bounds (clockwise) a subgraph $A_{\text {sub }} \subseteq A$ and b) no vertex in $V\left(A_{\text {sub }}\right) \backslash V\left(N_{A}\right)$ is incident to a c-edge. The subgraph $A_{\text {sub }}$ is 
representing the part of model $M$ already computed, whereas the residual graph of $A$ represents the part of $M$ which still has to be verified. For every middle set, we store this information in an array of triples $\left\langle A, N_{A}, \gamma\right\rangle$.

We define now valid mappings between combinatorial nooses and describe how partial solutions are stored in the dynamic programming. Then, we give the different DP-steps and finally verify the approach.

Valid partial solutions. For middle set $\operatorname{mid}(e)$ of the rooted sc-decomposition $\langle T, \mu, \pi\rangle$ of plane graph $G, N=N_{e}$ is the associated combinatorial noose in $G$ with face-vertex sequence of $F(N) \cup V(N)$ separating $G_{e}$ from the residual graph. Let $\mathfrak{N}$ denote the set of all combinatorial nooses of $A$ whose length is at most the length of $N$ and which bound (clockwise) a subgraph $A_{\text {sub }} \subseteq A$ such that no vertex in $V\left(A_{\text {sub }}\right) \backslash V\left(N_{A}\right)$ is an end-vertex of a c-edge. We now map $N$ to nooses $N_{A} \in \mathfrak{N}$, preserving the order. More precisely, we map vertices of $N$ to both vertices and faces of $A$. Therefore, we consider partitions of $V(N)=$ $V_{1}(N) \dot{\cup} V_{2}(N)$ where vertices in $V_{1}(N)$ are mapped to vertices of $V(A)$ and vertices in $V_{2}(N)$ to faces of $F(A)$.

We define a mapping $\gamma: V(N) \cup F(N) \rightarrow V(A) \cup F(A)$ relating $N$ to the combinatorial nooses in $\mathfrak{N}$. For every $N_{A} \in \mathfrak{N}$ on faces and vertices of set $F\left(N_{A}\right) \cup V\left(N_{A}\right)$ and for every partition $V_{1}(N) \dot{\cup} V_{2}(N)$ of $V(N)$, mapping $\gamma$ is valid $[\star]$ if

a) $\gamma$ restricted to $V_{1}(N)$ is a bijection to $V\left(N_{A}\right)$;

b) every $v \in V_{2}(N)$ and $f \in F(N)$ satisfy $\gamma(v) \in F\left(N_{A}\right)$ and $\gamma(f) \in F\left(N_{A}\right)$;

c) for every $v_{i} \in V(N)$ and subsequence $\left[f_{i-1}, v_{i}, f_{i}\right]$ of $N$ : if $v_{i} \in V_{2}(N)$, then face $\gamma\left(v_{i}\right)$ is equal to both $\gamma\left(f_{i-1}\right)$ and $\gamma\left(f_{i}\right)$, and if $v_{i} \in V_{1}(N)$, then vertex $\gamma\left(v_{i}\right)$ is incident to both $\gamma\left(f_{i-1}\right)$ and $\gamma\left(f_{i}\right)$; and

d) $A_{\text {sub }}$ is a minor of $G_{e}$ with respect to $\left.\left.a\right)-c\right)$.

We assign an array $\Psi_{e}$ to each $\operatorname{mid}(e)$ consisting of triples, where each triple $\left\langle A, N_{A}, \gamma\right\rangle$ represents a minor candidate $A$ together with a valid mapping $\gamma$ from a combinatorial noose $N$ corresponding to $\operatorname{mid}(e)$ to a combinatorial noose $N_{A} \in \mathfrak{N}$. The vertices and faces of $N$ are oriented clockwise around the drawing of $G_{e}$. Without loss of generality, we assume for every $\left\langle A, N_{A}, \gamma\right\rangle$ the orientation of $N_{A}$ to be clockwise around the drawing of subgraph $A_{\text {sub }}$ of $A$.

Step 0: Initializing the leaves. For every parent edge $e$ of a leaf $v$ of $T$, we initialize for every $A \in \mathcal{A}$ the valid mappings from the combinatorial noose bounding the edge $\mu(v)$ of $G$ to every combinatorial noose of length at most two in $A$ (clockwise bounding at most one edge of $A$ ).

Step 1a): Update process. We update the arrays of the middle sets bottomup in post-order manner from the leaves of $T$ to root $r$. During this updating process it is guaranteed that the 'local' solutions for each minor associated with a middle set of the sc-decomposition are combined into a 'global' solution for the overall graph $G$.

In each dynamic programming step, we compare the arrays of two middle sets $\operatorname{mid}(e)$ and $\operatorname{mid}(f)$ in order to create a new array assigned to the middle set 
$\operatorname{mid}(g)$, where $e, f$, and $g$ have a vertex of $T$ in common. From [13] we know that the combinatorial noose $N_{g}$ is formed by the symmetric difference of the combinatorial nooses $N_{e}, N_{f}$ and that $G_{g}=G_{e} \cup G_{f}$. In other words, we are ensured that if two solutions on $G_{e}$ and $G_{f}$ bounded by $N_{e}$ and $N_{f}$ fit together, then they form a new solution on $G_{g}$ bounded by $N_{g}$. We now determine when two solutions represented as tuples in the arrays $\Psi_{e}$ and $\Psi_{f}$ fit together. We update two triples $\left\langle A^{1}, N_{A}^{1}, \gamma_{1}\right\rangle \in \Psi_{e}$ and $\left\langle A^{2}, N_{A}^{2}, \gamma_{2}\right\rangle \in \Psi_{f}$ to a new triple in $\Psi_{g}$ if

- $A^{1}=A^{2}=: A \in \mathcal{A}$ and every edge of $A$ with no endpoint in $V\left(N_{A}^{1}\right) \cup V\left(N_{A}^{2}\right)$ is an m-edge;

- for every $x \in\left(V\left(N_{e}\right) \cup F\left(N_{e}\right)\right) \cap\left(V\left(N_{f}\right) \cup F\left(N_{f}\right)\right)$, we have $\gamma_{1}(x)=\gamma_{2}(x)$; and

- for the subgraph $A_{\text {sub }}^{1}$ of $A$ separated by $N_{A}^{1}$ and the subgraph $A_{\text {sub }}^{2}$ of $A$ separated by $N_{A}^{2}$, we have that $E\left(A_{\text {sub }}^{1}\right) \cap E\left(A_{\text {sub }}^{2}\right)=\emptyset$ and $V\left(A_{\text {sub }}^{1}\right) \cap V\left(A_{\text {sub }}^{2}\right) \subseteq$ $\left\{\gamma(v) \mid v \in V\left(N_{e}\right) \cap V\left(N_{f}\right)\right\}$.

That is, we only update solutions with the same graph $A$ and with the two nooses $N_{A}^{1}$ and $N_{A}^{2}$ bounding (clockwise) two edge-disjoint parts of $A$ and intersecting in a consecutive subsequence of both $N_{A}^{1}$ and $N_{A}^{2}$. If the two solutions on $N_{e}$ and $N_{f}$ fit together, we get a valid mapping $\gamma_{3}: N_{g} \rightarrow N_{A}^{3}$ to a noose $N_{A}^{3}$ of $A$ as follows:

- for every $x \in\left(V\left(N_{e}\right) \cup F\left(N_{e}\right)\right) \cap\left(V\left(N_{f}\right) \cup F\left(N_{f}\right)\right) \cap\left(V\left(N_{g}\right) \cup F\left(N_{g}\right)\right.$, ) we have $\gamma_{1}(x)=\gamma_{2}(x)=\gamma_{3}(x)$;

- for every $y \in\left(V\left(N_{e}\right) \cup F\left(N_{e}\right)\right) \backslash\left(V\left(N_{f}\right) \cup F\left(N_{f}\right)\right)$ we have $\gamma_{1}(y)=\gamma_{3}(y)$; and

- for every $z \in\left(V\left(N_{f}\right) \cup F\left(N_{f}\right)\right) \backslash\left(V\left(N_{e}\right) \cup F\left(N_{e}\right)\right)$ we have $\gamma_{2}(z)=\gamma_{3}(z)$.

We have that $\gamma_{3}$ is a valid mapping from $N_{g}$ to the combinatorial noose $N_{A}^{3}$ that bounds subgraph $A_{\text {sub }}^{3}=A_{\text {sub }}^{1} \cup A_{\text {sub }}^{2}$.

Step 1b): Post-processing. Before adding a triple $\left\langle A, N_{A}^{3}, \gamma_{3}\right\rangle$ to array $\Psi_{g}$, we need to manipulate $A$ so that $a$ ) it does not grow too big and $b$ ) it is suitable for future update operations. In $A$ restricted to subgraph $A_{\text {sub }}^{3}$, we contract all c-edges with at least one end-vertex not in $N_{A}^{3}$ in order to fulfill $a$ ). Concerning $b$ ), for every $B \in \mathcal{A}$ we check for all its nooses $N_{B}$ with $\left|V\left(N_{B}\right)\right|=\left|V\left(N_{A}^{3}\right)\right|$ if there is a bijection $\beta$ from $N_{A}^{3}$ to $N_{B}$ such that the following holds. If in a copy of $B$ we contract those c-edges which $i$ ) are in the subgraph counter-clockwise bounded by $N_{B}$ and $i i$ ) have at least one end-vertex not in $N_{B}$, then we obtain a 2-edge-colored graph isomorphic to $A$. We define $\delta=\gamma_{3} \circ \beta$ and we replace $\left\langle A, N_{A}^{3}, \gamma_{3}\right\rangle$ in array $\Psi_{g}$ by those triples $\left\langle B, N_{B}, \delta\right\rangle$ which validate properties $i$ ) and $i i)$.

Step 2: Termination. If, at some step, we have a solution where the entire minor $H$ is formed, we terminate the algorithm accepting. That is the case, if for some triple we have that $H \preceq A_{\text {sub }} \preceq A$ and $A_{\text {sub }}$ is bounded by $N_{A}$. We output model $M$ of $H$ in $G$ represented by this $A$ by reconstructing a solution top-down in $\langle T, \mu, \pi\rangle$. If at root $r$ no $A \in \mathcal{A}$ has been computed, we reject. 
We omit here the correctness proof and the running time of the algorithm and refer to the long version of this paper 2]. This completes the proof of Lemma 2.

Proof of Theorem 1. We put everything together by verifying Algorithm 1. We produce in time $\mathcal{O}\left(n^{2} \cdot \log n\right)$ a sc-decomposition of input graph $G$ [17. Next, either we can immediately compute a minor model of $G$ in time $\mathcal{O}\left(n^{2} \cdot \log n+h^{4}\right)$ (Proposition 6) or we run our 2-step-algorithm: we produce all majors of the minor pattern (Lemma 1) with Algorithm PRE-PROC [ $~ \star]$ in time $2^{\mathcal{O}(h)}$, and run partially embedded dynamic programming in time $2^{\mathcal{O}(h)} \cdot n$ (Lemma 2 ).

\section{Conclusions and Further Research}

In this paper we showed that Planar $H$-Minor Containment is solvable in time $2^{\mathcal{O}(h)} \cdot n+\mathcal{O}\left(n^{2} \cdot \log n\right)$ for a host graph on $n$ vertices and a pattern $H$ on $h$ vertices. That is, we showed that the problem can be solved in singleexponential time in $h$, significantly improving all previously known algorithms. Similar to 11, we can enumerate and count the number of models within the same time bounds.

Let us discuss some interesting avenues for further research concerning minor containment problems. First, it seems possible to solve in single-exponential time other variants of planar minor containment using our approach, like looking for a contraction minor, an induced minor, or a topological minor, as it has been recently done in [1] for general host graphs using completely different techniques. Also, it would be interesting to count the number of non-isomorphic models faster than just by enumerating models and removing isomorphic duplicates.

An important question is if, up to some assumption from complexity theory, the running time of our algorithm is tight. In other words, is there a $2^{o(h)}$. $n^{\mathcal{O}(1)}$ algorithm (i.e., a subexponential algorithm) solving PLANAR $H$-MINOR Containment or the existence of such an algorithm would imply the failure of, say, the Exponential Time Hypothesis? A first step could be to study the existence of subexponential algorithms when the pattern is restricted to be a $k$ outerplanar graph for some constant $k$, or any other subclass of planar graphs.

Conversely, single-exponential algorithms may exist for host graphs more general than planar graphs. The natural candidates are host graphs embeddable in an arbitrary surface. One possible approach could be to use the framework recently introduced in [29] to perform dynamic programming for graphs on surfaces. The main ingredient of this framework is a new type of branch decomposition of graphs on surfaces, called surface cut decomposition, which plays the role of sphere cut decompositions for planar graphs.

\section{References}

1. Adler, I., Dorn, F., Fomin, F.V., Sau, I., Thilikos, D.M.: Faster Parameterized Algorithms for Minor Containment. In: Kaplan, H. (ed.) SWAT 2010. LNCS, vol. 6139, pp. 322-333. Springer, Heidelberg (2010) 
2. Adler, I., Dorn, F., Fomin, F.V., Sau, I., Thilikos, D.M.: Fast Minor Testing in Planar Graphs (2010), http://users.uoa.gr/ sedthilk/papers/fastminorch.pdf

3. Alber, J., Bodlaender, H.L., Fernau, H., Kloks, T., Niedermeier, R.: Fixed parameter algorithms for dominating set and related problems on planar graphs. Algorithmica 33, 461-493 (2002)

4. Baker, B.S.: Approximation algorithms for NP-complete problems on planar graphs. Journal of the ACM 41, 153-180 (1994)

5. Demaine, E.D., Fomin, F.V., Hajiaghayi, M.T., Thilikos, D.M.: Subexponential parameterized algorithms on graphs of bounded genus and $H$-minor-free graphs. Journal of the ACM 52(6), 866-893 (2005)

6. Demaine, E.D., Hajiaghayi, M.: Bidimensionality. In: Kao, M.-Y. (ed.) Encyclopedia of Algorithms. Springer, Heidelberg (2008)

7. Demaine, E.D., Hajiaghayi, M.T.: Bidimensionality: new connections between FPT algorithms and PTASs. In: Proc. of the 16th Annual ACM-SIAM Symposium on Discrete Algorithms (SODA), pp. 590-601 (2005)

8. Demaine, E.D., Hajiaghayi, M.T., Kawarabayashi, K.i.: Algorithmic Graph Minor Theory: Decomposition, Approximation, and Coloring. In: Proc. of the 46th Annual IEEE Symposium on Foundations of Computer Science (FOCS), pp. 637-646 (2005)

9. Diestel, R.: Graph Theory, vol. 173. Springer, Heidelberg (2005)

10. Dinneen, M., Xiong, L.: The Feasibility and Use of a Minor Containment Algorithm. Computer Science Technical Reports 171, University of Auckland (2000)

11. Dorn, F.: Planar Subgraph Isomorphism Revisited. In: Proc. of the 27th International Symposium on Theoretical Aspects of Computer Science (STACS), pp. 263-274 (2010)

12. Dorn, F., Fomin, F.V., Thilikos, D.M.: Subexponential parameterized algorithms. Computer Science Review 2(1), 29-39 (2008)

13. Dorn, F., Penninkx, E., Bodlaender, H.L., Fomin, F.V.: Efficient exact algorithms on planar graphs: Exploiting sphere cut decompositions. Algorithmica (2009) (to appear)

14. Downey, R.G., Fellows, M.R.: Parameterized Complexity. Springer, Heidelberg (1999)

15. Fellows, M.R., Langston, M.A.: On search, decision and the efficiency of polynomial-time algorithms. J. Comp. Syst. Sc. 49, 769-779 (1994)

16. Garey, M.R., Johnson, D.S.: Computers and Intractability, A Guide to the Theory of NP-Completeness. W.H. Freeman and Company, New York (1979)

17. Gu, Q.-P., Tamaki, H.: Constant-factor approximations of branch-decomposition and largest grid minor of planar graphs in $O\left(n^{1+\varepsilon}\right)$ time. In: Dong, Y., Du, D.-Z., Ibarra, O. (eds.) ISAAC 2009. LNCS, vol. 5878, pp. 984-993. Springer, Heidelberg (2009)

18. Gu, Q.P., Tamaki, H.: Improved bound on the planar branchwidth with respect to the largest grid minor size. Technical Report SFU-CMPT-TR 2009-17, Simon Fraiser University (2009)

19. Hicks, I.V.: Branch decompositions and minor containment. Networks 43(1), 1-9 (2004)

20. Kawarabayashi, K.i., Reed, B.A.: Hadwiger's conjecture is decidable. In: Proc. of the 41st Annual ACM Symposium on Theory of Computing (STOC), pp. 445-454 (2009)

21. Kawarabayashi, K.i., Wollan, P.: A shorter proof of the Graph Minor Algorithm The Unique Linkage Theorem. In: Proc. of the 42st Annual ACM Symposium on Theory of Computing, STOC (to appear, 2010) 
22. Lipton, R.J., Tarjan, R.E.: Applications of a planar separator theorem. SIAM J. Comput. 9, 615-627 (1980)

23. Mohar, B., Thomassen, C.: Graphs on surfaces. John Hopkins University Press (2001)

24. Osthus, D., Prömel, H.J., Taraz, A.: On random planar graphs, the number of planar graphs and their triangulations. J. Comb. Theory, Ser. B 88(1), 119-134 (2003)

25. Reed, B.A., Li, Z.: Optimization and Recognition for $K_{5}$-minor Free Graphs in Linear Time. In: Proc. of the 8th Latin American Symposium on Theoretical Informatics (LATIN), pp. 206-215 (2008)

26. Robertson, N., Seymour, P.: Graph Minors. XIII. The Disjoint Paths Problem. J. Comb. Theory, Ser. B 63(1), 65-110 (1995)

27. Robertson, N., Seymour, P., Thomas, R.: Quickly excluding a planar graph. J. Comb. Theory, Ser. B 62(2), 323-348 (1994)

28. Robertson, N., Seymour, P.D.: Graph Minors. XX. Wagner's Conjecture. J. Comb. Theory, Ser. B 92(2), 325-357 (2004)

29. Rué, J., Sau, I., Thilikos, D.M.: Dynamic Programming for Graphs on Surfaces. In: Proc. of the 37th International Colloquium on Automata, Languages and Programming, ICALP (to appear, 2010), http://hal .archives-ouvertes.fr/inria-00443582

30. Seymour, P.D., Thomas, R.: Call routing and the ratcatcher. Combinatorica 14(2), 217-241 (1994)

31. Tutte, W.T.: A census of planar triangulations. Canadian Journal of Mathematics $14,21-38$ (1962) 